University of Nebraska - Lincoln

DigitalCommons@University of Nebraska - Lincoln

February 1990

\title{
Semiempirical determination of molecular motions associated with phonon modes in molecular crystals
}

K.M. White

University of Nebraska - Lincoln

Craig J. Eckhardt

University of Nebraska - Lincoln, ceckhardt1@unl.edu

Follow this and additional works at: https://digitalcommons.unl.edu/chemistryeckhardt

Part of the Chemistry Commons

White, K.M. and Eckhardt, Craig J., "Semiempirical determination of molecular motions associated with phonon modes in molecular crystals" (1990). Craig J. Eckhardt Publications. 19.

https://digitalcommons.unl.edu/chemistryeckhardt/19

This Article is brought to you for free and open access by the Published Research - Department of Chemistry at DigitalCommons@University of Nebraska - Lincoln. It has been accepted for inclusion in Craig J. Eckhardt Publications by an authorized administrator of DigitalCommons@University of Nebraska - Lincoln. 


\title{
Semiempirical determination of molecular motions associated with phonon modes in molecular crystals
}

\author{
K. M. White ${ }^{\text {a) }}$ and C. J. Eckhardt ${ }^{\text {b) }}$ \\ Department of Chemistry, University of Nebraska, Lincoln, Nebraska 68588-0304
}

(Received 11 August 1989; accepted 22 November 1989)

\begin{abstract}
An approach using piezomodulated Raman scattering is presented which permits determination of the molecular motions associated with the eigenmodes of molecular crystals. When coupled with a lattice dynamical calculation for the eigenmodes, the experiment can be used to validate the calculation. The case of 1,2,4,5-tetrabromobenzene is treated and it is shown that the phase relations of the piezomodulated Raman signals confirm the calculated eigenmodes.
\end{abstract}

\section{INTRODUCTION}

Knowledge of the microscopic mechanisms involved in solid-solid phase transitions in molecular crystals is requisite for the understanding, prediction, and eventual control of intermolecular interactions present in these systems. While x-ray crystallography has often provided accurate definitions of the structures of crystalline phases that are involved in a solid state phase transition, these time-averaged pictures can only suggest possible schemes that describe the dynamics of the transition. More definitive descriptions have been obtained from examination of the lattice dynamics of molecular crystals in which their low frequency lattice vibrations are characterized. Raman and infrared spectroscopy have been used extensively to identify these lattice phonon modes. In such spectra, the appearance or disappearance of vibrational bands in a molecular crystal over a particular temperature range may be evidence of a phase transition in the solid, as can changes in frequency or symmetry (or both) of the lattice phonon modes.

Raman and infrared spectroscopic studies, however, are unable to determine the actual molecular motions that take place during a phase transition. Even though these experiments have provided numerous assignments of frequencies and symmetries of phonon modes in molecular crystals, much less is known about the lattice mode eigenvectors which describe the motions the molecules in the crystal undergo during a vibration. Without resorting to somewhat less certain lattice-dynamics calculations, little more than symmetry assignments of these eigenvectors have been obtained experimentally. Determination of the lattice mode eigenvectors in molecular crystals is vital, since they play a key role in defining the microscopic mechanisms sought in solid-solid phase transitions and phonon-assisted solid state reactions.

Anharmonicity manifested by lattice vibrations is known to be responsible for solid state phase transitions. Therefore, an experimental approach that directly probes the coupling exhibited by these modes also has the potential for providing information regarding the molecular motions involved in the vibrations. Recently, piezomodulated Raman (PR) spectroscopy was shown to provide an experi-

\footnotetext{
a) Present address: 3M Company, St. Paul, Minnesota 55144-1000.

b) Author to whom correspondence should be addressed.
}

mental measure of coupling by the significantly anharmonic $21.5 \mathrm{~cm}^{-1}$ lattice mode in 1,2,4,5-tetrabromobenzene (TBB). ${ }^{1}$ Although the PR responses of the other Raman active lattice modes in TBB were observed to be smaller, further investigation shows that for a given stress, the phases of the derivative-like PR bands are not all the same. This experimental information, considered jointly with the $\mathbf{P R}$ theory ${ }^{2}$ and a recent determination of the lattice mode symmetries in $\mathrm{TBB},{ }^{3}$ indicates that it is possible to extract experimental evidence for the motions of molecules in crystals from PR data.

The molecular motions associated with a given phonon have additional importance to chemical reactions in solids. Specific vibrational modes have been shown to be involved with solid state reactions. ${ }^{4}$ While the frequency of the phonon modes may be identified, much greater mechanistic understanding would be obtained if the actual molecular motions associated with these modes could be determined. This knowledge would permit detailed elaboration of the actual molecular motions involved with a solid state reaction.

Theoretical and experimental details pertaining to the PR experiment will be outlined first, followed by a presentation of the results observed for the Raman active lattice modes in TBB. An interpretation of the data will be made in terms of the motions allowed for these modes in the TBB crystal. Finally, the potential of PR spectroscopy as a powerful tool in the determination of the microscopic aspects of lattice dynamics in molecular crystals will be considered.

\section{THEORY AND EXPERIMENT}

In PR spectroscopy, a modulated stress is applied to a crystal and the inelastically scattered light is detected synchronously with the modulation. A hypothetical example illustrates (Fig. 1) the PR spectral response. Consider a Raman active lattice mode of frequency $\omega_{0}$. In addition, suppose that a tensile uniaxial stress applied in some given direction results in a decrease of the mode frequency to $\omega_{-}$, while a compressive stress causes the frequency to increase to $\omega_{+}$. If the tensile and compressive stresses are applied to the crystal in a periodic fashion, then synchronous detection of the Raman scattered light will provide a measurement of the intensity difference between the $\omega_{-}$and $\omega_{+}$Raman bands at the frequency setting of the monochromator, $\omega$, 


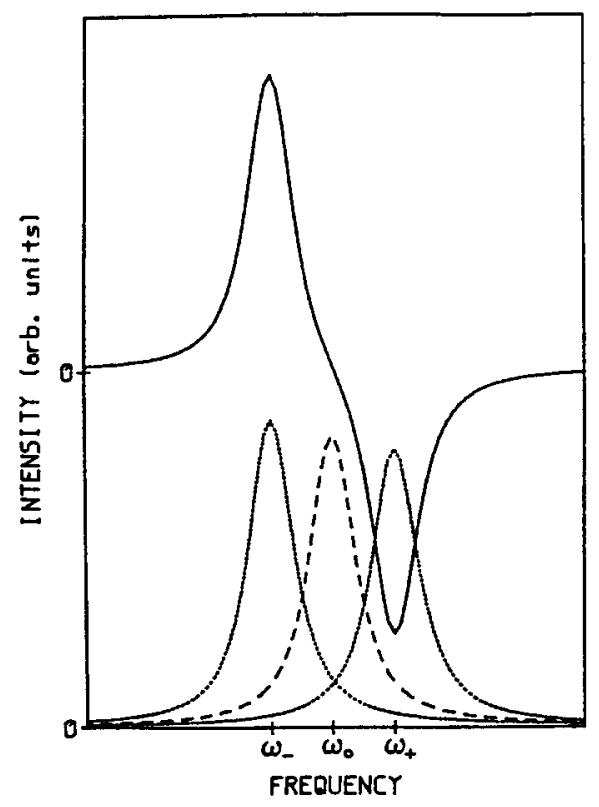

FIG. 1. Hypothetical "positive" PR band ( solid line) for mode $\omega_{0}$ (dashed line) under tensile $\left(\omega_{-}\right)$and compressive $\left(\omega_{+}\right)$stresses (dotted lines).

$$
\Delta I(\omega)=I_{\omega_{-}}(\omega)-I_{\omega_{+}}(\omega) .
$$

Using a Lorentzian model to describe the Raman response of the vibrational mode, the resulting bandshapes of the Raman and piezomodulated Raman signals are shown in Fig. 1.

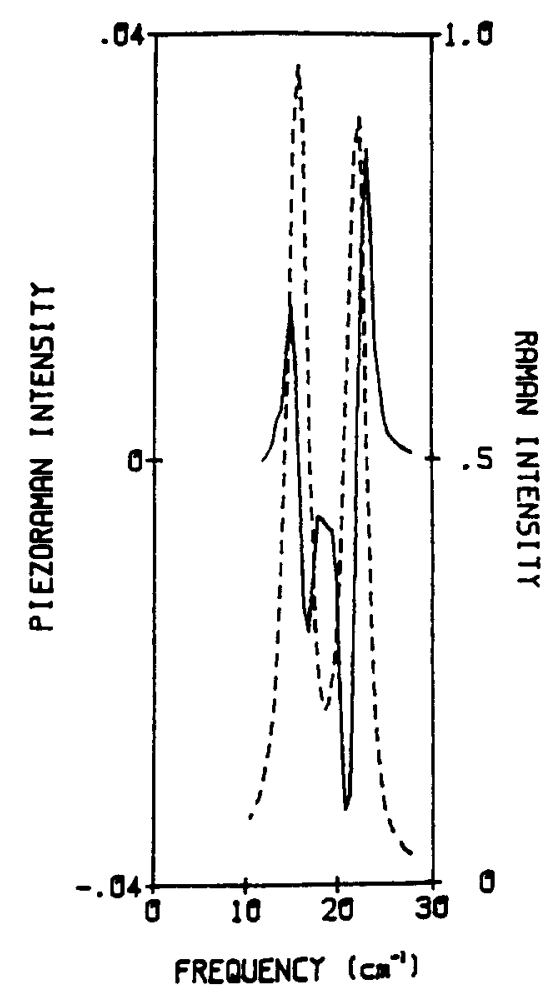

FIG. 2. PR spectrum (dashed line) of the 15.5 and $21.5 \mathrm{~cm}^{-1}$ lattice vibrations in TBB along with the corresponding Raman spectrum. The [001] stress employed in this particular PR spectrum provides examples of both positive and negative PR bands in TBB.
The PR response is similar but not equivalent to the frequency derivative of the Raman band itself. Instead, it can be shown by making use of the one phonon oscillator approximation of Luty and Eckhardt ${ }^{2}$ that for small strains, the intensity expression above is actually the strain derivative of the Raman response of the vibrational mode.

The hypothetical example above assumed that a compressive stress resulted in an increase in mode frequency. For lattice modes in molecular crystals, this has been the typical response when hydrostatic compressive stresses have been applied in previous Raman experiments. ${ }^{5-7}$ However, this need not be the case when uniaxial stresses are employed. The nature of the phase information obtained from the PR spectra indicates that a positive-type $P R$ band, in which the band goes positive and then negative when scanning from low to high energy (see Fig. 1), corresponds to a mode in which a compressive stress increases the vibrational frequency, while a tensile stress results in a decrease in frequency. On the other hand, a mode giving rise to a negative-type PR band displays the opposite behavior: a decrease in vibrational frequency under compressive stress and an increase under tensile stress. Thus, the uniaxial aspect of the PR experiment yields directional information pertaining to intermolecular interactions and their resulting vibrational modes in the crystal based on the phases of the PR bands alone.

Preparation of the TBB crystal samples is described elsewhere, ${ }^{1,3}$ as is the apparatus used in the piezomodulated Raman experiment. ${ }^{1,3,8}$ The crystals, which have space group symmetry $P 2_{1} / a\left({ }^{5} C_{2 h}\right)$, are columnar along $c$ with form $\{110\}\}^{9,10}$ The crystals were glued in the appropriate stress configuration between a rigid stainless steel bar and a piezoceramic bar secured at one end. Stresses were induced by applying a high sinusoidal voltage across the piezoceramic bar. The experiments were performed in two distinct stress configurations: (1) the stress normal to (110) (direction denoted [110]*), and (2) the stress direction of [001]. The spectral measurements employed the $5145 \AA$ line of an argon ion laser operating at $55 \mathrm{~mW}$ and scanned the range from 8 to $60 \mathrm{~cm}^{-1}$ thereby encompassing all of the TBB Raman active lattice modes. Various polarization geometries were used so that all of the modes could be observed for a given stress.

\section{RESULTS AND DISCUSSION}

A piezomodulated Raman spectrum of the 15.5 and $21.5 \mathrm{~cm}^{-1}$ lattice vibrations in TBB is shown in Fig. 2 along with the corresponding Raman spectrum. The [001] stress employed in this PR spectrum gives rise to a positive PR band for the $15.5 \mathrm{~cm}^{-1}$ mode and a negative $P R$ band for the $21.5 \mathrm{~cm}^{-1}$ phonon. Further examination of the phases of all of the PR bands measured in TBB in the lattice vibration energy region shows that the 21.5 and $39 \mathrm{~cm}^{-1}$ modes increase in frequency under a compressive stress in the [110]* direction and decrease for a compressive stress along [001]. The remaining Raman active lattice modes at 15.5, 41.5, 43, and $47.5 \mathrm{~cm}^{-1}$ show the opposite behavior.

Tabulation of the PR data (Table I) reveals a clear pattern. Its explanation can be obtained from a consideration of the strains induced in the TBB crystals by the $[110]^{*}$ and 
TABLE I. Phases of the piezomodulated Raman bands corresponding to the lattice modes in TBB. ${ }^{\text {a }}$

\begin{tabular}{cccc}
\hline $\begin{array}{c}\text { Mode } \\
\text { frequency } \\
\left(\mathrm{cm}^{-1}\right)\end{array}$ & $\begin{array}{c}\text { Mode } \\
\text { symmetry }\end{array}$ & {$[110]^{*}$} & Stress \\
\hline 15.5 & $A_{g}$ & - & {$[001]$} \\
21.5 & $B_{g}$ & + & + \\
39. & $B_{g}$ & + & - \\
41.5 & $A_{g}$ & - & + \\
43. & $B_{g}$ & - & + \\
47.5 & $B_{g}$ & - & + \\
\hline
\end{tabular}

${ }^{2}$ Positive phase indicates an increase in frequency upon compressive stress and negative phase indicates a decrease in frequency upon compressive stress.

[001] stresses and from the symmetries of the allowed molecular motions for the six Raman active lattice vibrations in TBB.

The strains e produced in a crystal under a given stress $\sigma$ can be determined using the compliance tensor for the crystal S:

$$
\mathbf{e}=\mathbf{S} \boldsymbol{\sigma} .
$$

In this expression, the macroscopically defined stresses and strains are expressed in matrix notation, with e and $\sigma$ described by six-dimensional column vectors and $S$ by a $6 \times 6$ matrix. For a crystal of any given symmetry, the number of independent, nonzero elements of the compliance tensor can be determined, allowing one to derive the types of strains induced in the crystal by the application of specified stresses. ${ }^{11}$ In addition, the symmetries of the strains can be determined with the use of the character table that corresponds to the point group symmetry of the crystal. ${ }^{12}$

Owing to its monoclinic symmetry, a stress in TBB along [110]* is found to give rise to shear strains of $B_{g}$ symmetry in the $a^{*} b$ and $b c$ crystallographic planes. On the other hand, the [001] stress will only produce strains of $A_{g}$ symmetry along the $a^{*}, b$, and $c$ axes and a shear strain in the $a^{*} c$ plane. Thus, the two stress directions used in the PR experiments result in two mutually exclusive sets of strains in TBB having different symmetries.

Because the elements along the diagonal of the compliance tensor in a molecular crystal are generally larger than the off-diagonal elements, it is expected that the greatest displacements in the TBB crystal will be in the same direction as the applied stresses. However, the presence of the additional strains must also be taken into account in the data analysis. Moreover, it should be understood that by attaching the crystal to the sample mount, stresses other than the one supplied by the piezoceramic are present in the experiment. However, these are actually surface stresses and their influence was minimized by measuring the PR spectra from the crystal bulk.

The TBB molecules are located at sites of inversion symmetry in the crystal unit cell, therefore only librational motions of the molecules contribute to the Raman active lattice modes. These motions involve small, rotational displacements of the molecules about their three principal axes of inertia, with the two molecules in the unit cell having their movements in-phase for the $\boldsymbol{A}_{g}$ modes and out of phase for the $B_{\mathrm{g}}$ modes. The resulting six degrees of freedom account for the six Raman active lattice modes in TBB.

While the librational motions for a given mode seldom involve pure rotations about a single inertial axis of the molecules, the eigenvector is often found to describe predominant motion about one particular axis. If this is taken to be the case for each of the six Raman active lattice modes in TBB, then the modes can be paired off as in-phase and out-ofphase librations about the axis normal to the molecular plane and about each of the two inertial axes contained in the plane.

An explanation of the pattern in Table I can now be made. The compressive $[110]^{*}$ stress, as shown in Fig. 3, causes the TBB molecules lying nearly in the $a^{*} b$ plane to be forced closer together in a side-by-side fashion. This means the intermolecular contacts on the perimeter of the molecules will interact more strongly, resulting in larger in-plane forces between them. This type of interaction would mostly affect a primarily in-plane libration by increasing its energy and thus its vibrational frequency. Since two in-plane librational modes are predicted, one each of $A_{g}$ and $B_{g}$ symmetry, there should be two corresponding positive bands in the piezomodulated spectrum employing a [110]* stress. Indeed, the $B_{g}$ mode at $21.5 \mathrm{~cm}^{-1}$ and the $A_{g}$ mode at 39 $\mathrm{cm}^{-1}$ display such a response in the $[110]^{*}$ stress PR spectra, indicating that their associated molecular librational motions are principally in-plane.

The compressive [001] stress (Fig. 4) forces TBB molecules in neighboring unit cells together along the stacking direction ( $c$ axis) of the lattice. For these displacements, out-of-plane intermolecular interactions will be increased, thus affecting the primarily out-of-plane librational modes by raising their frequencies. Consequently, four positive bands, two corresponding to $A_{g}$ active modes and the other two to $B_{g}$ active modes, would be expected in PR spectra

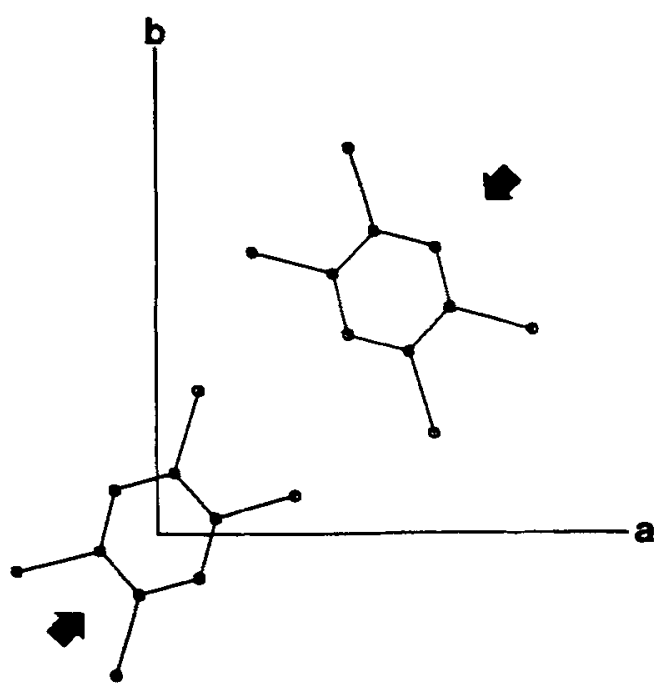

FIG. 3. Projection of $[110]^{*}$ stress for $\beta$-TBB, as viewed down the $c$ axis. 


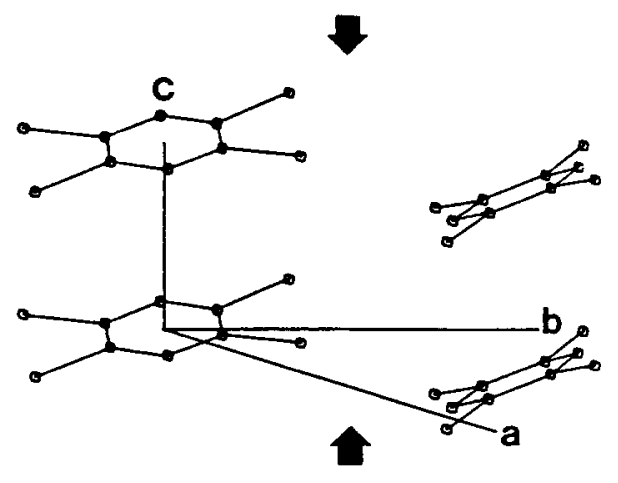

FIG. 4. (110) projection of [001] stress for $\beta$-TBB.

involving a [001] stress. Again, this is observed in the data. The $A_{g}$ modes at 15.5 and $41.5 \mathrm{~cm}^{-1}$ and the $B_{g}$ modes at 43 and $47.5 \mathrm{~cm}^{-1}$ are seen to have positive bands in these PR spectra. Therefore, their corresponding molecular librational motions are predominantly out of plane, as determined from the PR data.

A lattice-dynamics calculation that has been performed for $\mathrm{TBB}^{3}$ generated a calculated crystal potential energy that was capable of producing eigenvectors for the lattice modes that are consistent with the molecular motions determined from the piezomodulated Raman data. For the mode of lowest energy, the calculation indicated that the $15.5 \mathrm{~cm}^{-1} A_{g}$ mode is principally a libration about an axis lying in the molecular plane, which is in accordance with the phase information obtained from its PR bands. On the other hand, the $21.5 \mathrm{~cm}^{-1} B_{g}$ mode was found to have a large component of rotation about the axis normal to the molecular plane and this agrees with the interpretation of the PR data as well. The other four eigenvectors that have been calculated for TBB are also consistent with the PR results. The $41.5 \mathrm{~cm}^{-1}$ $A_{g}$ mode and the 43 and $47.5 \mathrm{~cm}^{-1} B_{g}$ modes, whose eigenvectors denote librations primarily about axes in the TBB molecular plane, all had positive PR bands in the [001] stressed spectrum. The $39 \mathrm{~cm}^{-1} A_{\mathrm{g}}$ mode, which exhibited a positive PR band in the [110]* stressed spectrum, is associated ${ }^{3}$ with the mode calculated at $40.6 \mathrm{~cm}^{-1}$ because its eigenvector corresponded to an in-phase libration about an axis normal to the molecular plane. This demonstrates that PR spectroscopy can be used in the assignment of lattice modes by comparing the molecular motions deduced from the PR spectra of the vibration to be assigned to the eigenvectors calculated for the various modes.

The question remains of why the phase of the PR band for each of the lattice vibrations reverses when going from a $[110]^{*}$ stressed spectrum to a [001] stressed spectrum, or vice versa. The answer is found by considering all possible strains induced in the crystal for these two stress directions. In the above discussion, only strains in the same direction as the applied stresses were used to explain the observed phases of the PR bands. However, for the case of the [110 ${ }^{*}$ stress, a shear strain in the $b c$ crystallographic plane also exists, while for the [001] stress, linear strains along the $a^{*}$ and $b$ axes and a shear strain in the $a^{*} c$ plane are also present. It is readily apparent, particularly for the additional extensive strains that can occur perpendicular to a [001] compressive stress, that the PR response of a given mode for one stress may be $180^{\circ}$ out of phase from its response for the perpendicular stress.

It should also be remembered that the [110]* and [001] stresses give rise to mutually exclusive sets of strains which are of different symmetries. Thus, the PR experiments employing the [110]* stress result in coupling of the Raman active modes of like symmetry, while the [001] stress enhances coupling of Raman modes of different $\left(A_{g}\right.$ and $\left.B_{g}\right)$ symmetry. ${ }^{2}$

\section{CONCLUSION}

The PR experiment, by directly probing the molecular motions that constitute the lattice vibrations, has been shown to provide an effective test for the validity of the eigenvectors obtained from a lattice-dynamics calculation. In so doing, it can also determine the reliability of the crystal potential energy in a new and more accurate fashion. It has always been possible to test eigenvectors in a more indirect manner by comparing calculated and observed Raman intensities, ${ }^{13-16}$ but this is extremely difficult in practice and quite unsatisfactory.

The foregoing interpretation of the phase information obtained from the PR spectra of the lattice modes in TBB is significant because it demonstrates that piezomodulated Raman spectroscopy can now be used as a direct, experimental probe for the determination of the molecular motions described by the eigenvectors of lattice vibrations in molecular crystals. Previously, only normal coordinate analyses and lattice-dynamics calculations were able to provide this information, and their success depended on the reliability of the force field or the potential energy derived for the calculations. They thus remained calculative determinations untested by experiment. Now, piezomodulated Raman spectroscopy can make use of a large number of stress directions to produce an experimental description of the vibrational motions which, in turn, may be used to confirm previous calculations or used to improve upon the potential energy functions derived for the calculations. Indeed, this was one of the major goals of developing this spectroscopy.

Major assumptions about the intermolecular interactions in TBB crystals were employed in the analysis given above and the description is necessarily phenomenological. Nevertheless, it demonstrates the potential of the technique and shows how the combination of PR studies and latticedynamics calculations can be used to determine the actual motions of the molecules in the lattice. Since the spectroscopy does probe intermolecular interactions in crystals, it will then be able to improve and refine the initial assumptions made for these forces and potential energies. Thus, piezomodulated Raman spectroscopy provides the first experimental means for the determination of eigenmode motions of molecules in molecular crystals. 
${ }^{1}$ K. M. White and C. J. Eckhardt, Phys. Rev. Lett. 59, 574 (1987).

${ }^{2}$ T. Luty and C. J. Eckhardt, J. Chem. Phys. 82, 1515 (1985).

${ }^{3}$ K. M. White and C. J. Eckhardt, J. Chem. Phys. 90, 4709 (1989).

${ }^{4}$ P. N. Prasad and J. Swiatkiewicz, Mol. Cryst. Liq. Cryst. 93, 25 (1983).

${ }^{5}$ B. A. Weinstein and R. Zallen, in Light Scattering in Solids IV: Electronic Scattering, Spin Effects, SERS, and Morphic Effects, Topics in Applied Physics, Vol. 54, edited by M. Cardona and G. Guntherodt (Springer, Berlin, 1984), p. 463.

${ }^{6}$ C. Carlone, N. K. Hota, H. J. Stolz, M. Elbert, and H. D. Hochheimer, J. Chem. Phys. 75, 3220 (1981).

${ }^{7}$ D. A. Dows, L. Hsu, S. S. Mitra, O. Brafman, M. Hayek, W. B. Daniels, and R. K. Crawford, Chem. Phys. Lett. 22, 595 (1973).
${ }^{8}$ K. M. White and C. J. Eckhardt (in preparation).

${ }^{9}$ B. Pasquier and N. Le Calve, J. Raman Spectrosc. 6, 155 (1977).

${ }^{10}$ G. Gafner and F. H. Herbstein, Acta Crystallogr. 13, 706 (1960)

${ }^{11}$ J. F. Nye, Physical Properties of Crystals (Oxford University, New York, 1985).

${ }^{12}$ S. Bhagavantam, Crystal Symmetry and Physical Properties (Academic, New York, 1966).

${ }^{13}$ K. M. White, K.-H. Brose, and C. J. Eckhardt, J. Chem. Phys. 85, 5551 (1986).

${ }^{14}$ T. Luty, A. Mierzejewski, and R. W. Munn, Chem. Phys. 29, 353 (1978)

${ }^{15}$ E. Burgos, H. Bonadeo, and E. D'Alessio, J. Chem. Phys. 63, 38 (1975).

${ }^{16}$ J. W. Arthur and G. A. Mackenzie, J. Raman Spectrosc. 4, 353 (1976). 